\title{
Comparative Study of PEGASIS Protocols in Wireless Sensor Network
}

\author{
Hetal Rana \\ M.E.I.T(pursuing) \\ Thakur College Of \\ Engineering And Technology \\ University of Mumbai
}

\author{
Sangeeta Vhatkar \\ Assistant Professor \\ Thakur College Of \\ Engineering And Technology \\ University of Mumbai
}

\author{
Mohommad Atique \\ Associate Professor \\ P.G. Department of Computer \\ Science S.G.B.A.U, \\ Amravati
}

\begin{abstract}
The area of Wireless Sensor Networks (WSNs) is one of the fast growing and emerging field in the scientific and engineering world. It is an ad-hoc network that consists of small nodes with sensing, computing and communicating wireless abilities. These sensor nodes are densely deployed in the sensor field environments. The environment can be an Information Technological framework, a physical world, or a biological system.The main objective of WSN is to sense the crucial information from the environment depending on the type of application for which it is deployed and send this information to its Base Station (BS) so that it can take corrective actions. These Sensor Nodes communicate with each other via various Routing Protocols. Protocols in WSNs are broadly classified as Flat, Hierarchical and Location Based routing protocols. This paper presentshierarchical routing protocol, Power Efficient Gathering in Sensor Information Systems (PEGASIS) and a comparative study on various versions of PEGASIS protocols.
\end{abstract}

Keywords:WSN, MANET, LEACH, PEGASIS, EEPB, PEGASIS-ANT, H-PEGASIS, PDCH, IEEPB.

\section{Introduction}

A WSN consists of hundreds and thousands of Sensor nodes. These nodes have ability to monitor physical or environmental conditions, such as temperature, sound, pressure and communicate this information wirelessly with each other or directly to the BS.WSN are expected to be the solutions to many applications such as, military, area monitoring, health monitoring, industry monitoring and many more.

Recent technological advances in the field of WSN, Micro Electronic Mechanical systems(MEMS) and Wireless Communication Technologies have enabled the development of tiny, low cost, low power, multifunctional smart sensor nodes.

These sensor nodes comprises of sensing, processing, transmission, mobilizer, position finding system and power units (some of these components are optional like the mobilizer). The figure shows the communication architecture of a WSN. Sensor nodes are usually scattered in a sensor field, which is an area where the sensor nodes are deployed. Sensor nodes coordinate among themselves to produce high-quality information about the physical environment. The decision of each sensor node is determined by its mission, the information it currently has and its knowledge of its computing, communication, and energy resources. Each of these scattered sensor nodes has the capability to collect and route data either to other sensors or back to an external base station(s). A base-station may be a fixed node or a mobile node capable of connecting the sensor network to an existing communications infrastructure or to the Internet where a user can have access to the reported data[1].

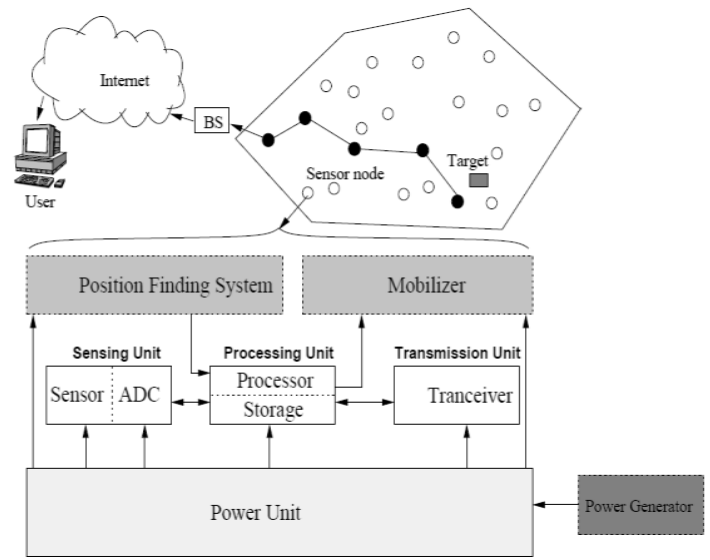

Fig 1: Components of WSN Node[1] 
Routing the information in WSN is very challenging due to its inherent characteristics that distinguish these networks from other wireless networks such as Mobile Ad-hoc Networks (MANET) or Cellular networks.

First, number of sensor nodes are enormous in WSN hence Global Addressing scheme cannot be deployed. Thus a traditional IP protocols cannot be applied to WSN [12].

Additionally, all the application of WSN, unlike traditional communication network requires the flow of sensed data from multiple sources to the BS. Thus here typical topologies of Communication Networks (bus, ring, peer to peer, multi-cast) cannot be implemented [2].

Following to that, all the sensor nodes are tightly constrained in the terms of energy, processing and storing capabilities, which may require careful storage management.

Succeeding that, nodes in WSN are expected to be stationary after the deployment except a few of the nodes, whereasin traditional wireless network the nodes are free and mobile which results unpredictable frequent topological changes[12].

Subsequently, all sensor networks are application specific, i.e. the design requirements for each network changes as per the application.

Finally, the data that is collected by the sensor nodes are dependent on common phenomena; hence there is high probability of data redundancy.

Due to such differences, there are various varieties of algorithms proposed for routing data in WSN.

The main issue in WSN is to identify the sensor node. Once nodes are identified, routing protocols are in charge of constructing and maintaining routes between distant nodes. The different ways in which routing protocols operate make them appropriate for certain applications [2].

Depending on the basis of network structure, Routing protocols are classified as Flat, Hierarchical and location based Routing protocols.Routing protocols can also be classified on the basis network operations such as Query Based, Negotiation Based, QOS based and coherent based. The figure shows the hierarchy of WSN routing protocols [1].

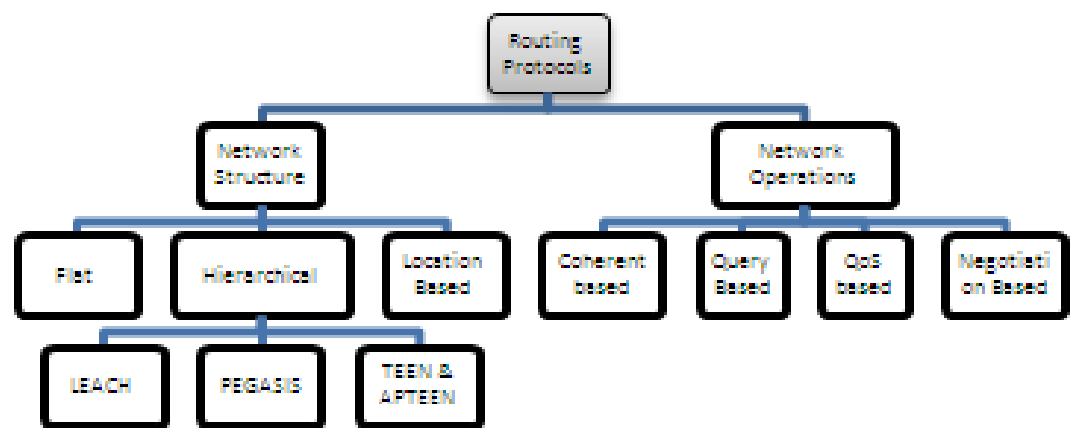

Fig 2: Hierarchy of Routing Protocols In WSN.

In this article we concentrate on Hierarchical Routing protocols, types of Hierarchical routing protocols, PEGASIS protocols and comparative study on various versions of PEGASIS protocols.

\section{Hierarchical Routing protocols[1].}

Hierarchical Routing Protocols (HRP) are also known as cluster based routing protocols that were originally proposed in wire line networks. They are well known techniques that have special advantages such as scalability and efficient communication. The concept of HRP is used to perform efficient routing in WSN. Such model breaks the whole network in to various layers of clusters.

Here nodes are grouped in to clusters with each cluster having a Cluster Head(CH)[3]. This $\mathrm{CH}$ is responsible, for routing the sensed information from the cluster to the BS. The data is transferred from the lower cluster to the upper cluster and so on. This forms a Hierarchical structure. In such structure the data is first aggregated in the cluster at lower level and then it sent to the higher level cluster to its $\mathrm{CH}$. As it moves from the lower level Cluster to the higher level cluster, it has to travel great distances, which requires high speed data transfer sensor nodes.

This model is better than multi-hop model because firstly, it has less latency than multi-hop model and secondly, in this model every $\mathrm{CH}$ performs the data aggregation, whereas in multi-hop model every node performs data aggregation, hence this is more suitable for time critical applications. The Fig 3 shows the basic structure of HRPs. 


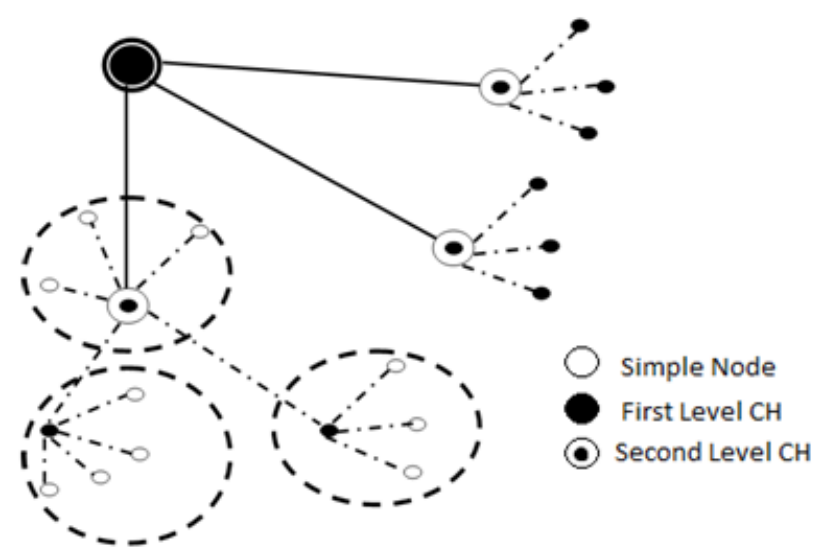

Fig 3: Structure of HRP [3].

\section{History of PEGASIS protocol}

As proposed in [4] Low Energy Adaptive Clustering Hierarchy (LEACH) is the first cluster based routing protocol that uses randomization to distribute the energy load evenly among the sensors in the network. Here the nodes organize themselves into local clusters, with one node as $\mathrm{CH}$. Also LEACH performs local data fusion in order to compress the data collected by the clusters before sending it to the BS, thus reducing energy dissipation and enhances system lifetime. Because of randomization of $\mathrm{CH}$, chosen to transmit the data to BS, LEACH achieves a factor of 8 improvement compared to direct transmissions as measured in terms of when the nodes die. Also It is not applicable to networks deployed in large regions. The idea of dynamic clustering brings extra overhead to each $\mathrm{CH}$. And lastly the protocol assumes that all nodes begin with the same amount of energy capacity in each election round, wasting lots of energy. The figure shows the illustration of LEACH protocol.

The article [6] PEGASIS is a near optimal chain-based routing protocol that is an improvement over LEACH.

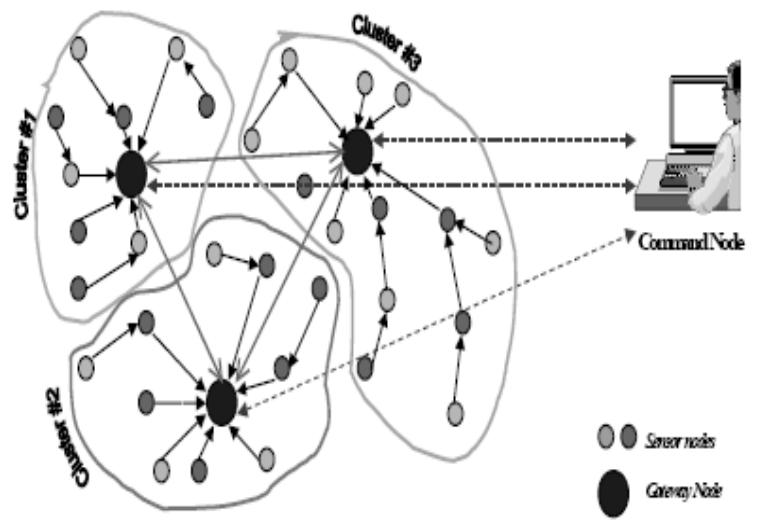

Fig 4: Illustration of LEACH [5]

\section{PEGASIS Protocol}

The main idea in PEGASIS protocol is for node to receive from and transmit to close neighbors and take turns for being the leader for transmission of data to BS. This approach distributes the energy load evenly among the sensor nodes. The nodes randomly placed in the field, organize themselves in the form of chain using greedy algorithm. Alternatively, BS computes this chain and broadcasts it to all the nodes.

Fig 5: shows node 0 and node 1 connecting to node 3 , node 3 connecting to node 1 and node 1 connecting to node 2 . When a node dies, the chain is constructed in the same manner to bypass the dead node.

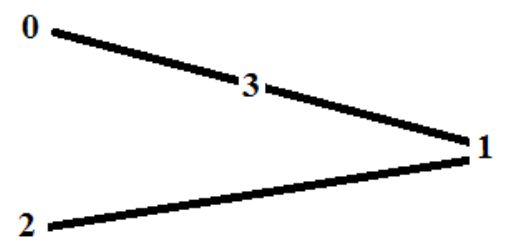

Fig 5: Chain formation 
For data gathering, each node receives the data from one neighbor, fuses its own data and transmits it to the next node in the chain. In a given round, a simple token passing approach is initiated by the leader to start the data transmission from the ends of the chain. Here the cost is very less because the size of the token is very small.

The fig 6: shows node $\mathrm{C} 2$ as the leader. It passes the token to $\mathrm{C} 0 . \mathrm{C} 0$ sends its data to $\mathrm{C} 1$. $\mathrm{C} 1$ fuses its own data with $\mathrm{C} 0$ 's data and sends it to leader $\mathrm{C} 2$. C2 then transmits the token to $\mathrm{C} 4$. C4 sends its data to C3. C3 fuses its data with C4's data and then transmits it to leader $\mathrm{C} 2$.

$\mathrm{C} 2$ waits to receive data from both the neighbors and then it fuses its data to neighbor's data. The leader then transmits only one message to the BS.

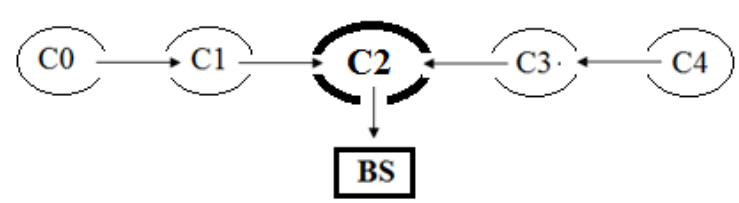

Fig 6: Token Passing

Thus in PEGASIS, each node receives and transmits one packet in each round and be the leader at least once in $\mathrm{n}$ rounds ( $\mathrm{n}$ are no of nodes).

PEGASIS improves on LEACH by saving energy at following stages.

First, in the local gathering, the distance that most of the nodes transmits is much less as compares to $\mathrm{CH}$ in LEACH. Second, the leader receives at most only two messages from the neighbors which is not in the case of LEACH (in the network of 100 nodes, it receives 20 messages if there are 20 nodes per cluster). Finally, one node transmits the message to the BS in each round of communication.

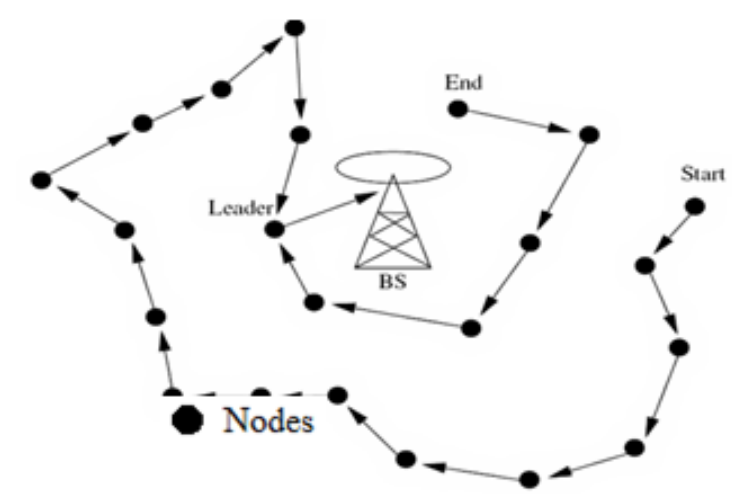

Fig 7: Illustration of PEGASIS Protocol

PEGASIS protocol has its major applications in environment monitoring[11]. The nodes sense various environmental factors such as temperature, humidity, pressure, etc. Each node fuses its sensed data with the adjacent node. The $\mathrm{CH}$ finally has all the sensed data, which it then sends to the BS.PEGASIS protocol has its main application in characterizing and monitoring the quality of environment,

\section{Types of PEGASIS protocols}

Though PEGASIS protocol has its advantages over LEACH protocol, it still had certain deficiencies. The below described protocols are various versions of PEGASIS that are designed to overcome those deficiencies. Each protocol takes into consideration unique factors and proposes its different version.

Energy Efficient PEGASIS Based (EEPB)is an enhanced PEGASIS algorithm [7] in WSN. As in PEGASIS greedy algorithm is used to form the data chain, it can result in communication distance between two sensors being too long. Thus the sensors consume more energy in transmitting the data and die early. In the chaining process, a node will consider the average distance of which the chain is formed. This distance is known as thresh distance. If the distance from the closest node to the upstream node is longer than thresh distance, the closest node is the "far node". If the closest node joins the chain, it will become "long chain". EB-PEGASIS avoids this phenomena using distance threshold. It not only saves energy on threshold, but also balances the energy consumption of all sensor nodes. 
ThePEGASIS-ANT [8] protocol uses ANT colony algorithm rather than greedy algorithm to construct the data chain. This helps to achieve global optimization. It forms the chain that makes the path more even-distributed and reduces the transmission distance. It also balances the energy consumption between the nodes. In each round of transmission, on the basis of current energy of each node the leader is selected that directly communicates with the BS. This algorithm has prolonged network lifetime.

H-PEGASIS [3] is an extended version of PEGASIS protocol.It was introduced with the objective of decreasing the delay of transmission packets to the BS. It proposes a solution to data gathering problems by considering energy X delay metrics. In order to reduce delay, simultaneous data messages are transmitted. To avoid collisions, signal coding is implemented e.g CDMA to avoid signal interference, only spatially separated nodes are allowed transmit data at the same time. With CDMA capable nodes, the chain forms the tree like hierarchy and each selected node transmit the data to the node of upper hierarchy. This ensures parallel data transmission and reduces the delay significantly.

PEGASIS with double Cluster Head(PDCH)[9] balances load of every node and increase network lifetime. Generally PEGASIS protocol uses one $\mathrm{CH}$ that communicates with the BS. Here instead of one double $\mathrm{CH}$ are used in a single chain and is given a hierarchical structure so that long chaining is avoided. PDCH outperforms PEGASIS by eliminating dynamic cluster formation, reducing the distance between nodes, reducing the number of messages sent to and from other nodes and using only one transmission to BS per round. As the energy load is distributed among the nodes, the network lifetime increases and so does the quality of network.

Improved Energy Efficient PEGASIS Based (IEEPB)[10] protocol, overcomes the deficiencies of EEPB. When EEPB builds a chain, the threshold adopted is uncertain and complex to determine. This results in the formation of "long chain". Also, when EEPB selects the leader, it ignores the node energy and the distance between the BS and node that optimizes the selection of leader. Based on this, IEEPB compares the distance between two nodes twice and finds the shortest path to link two adjacent nodes. The chain construction is simplified such that formation of "long chain" is avoided. Also while selecting the leader, IEEPB considers the node's energy, distance between the BS and the node, normalizes these twofactors and assigns different weight co-efficient to them. Finally the node with the minimum weight becomes the leader.IEEPB has higher energy efficiency and hence longer network lifetime.

\section{Conclusion and future work}

From the protocols reviewed, though PEGASIS protocol outperforms LEACH, it has certain drawbacks. As it uses greedy algorithm for formation of data chain, it results in the inevitable Long Chain thus consuming more energy due to which nodes die early. EEPB protocol tries to overcome the drawbacks of PEGASIS by using distance threshold. But it has more other deficiencies' as uncertain threshold leads to formation of LL. Also it ignores the node energy and distance between nodes and BS while selecting the leader. IEEPB overcomes these drawbacks by taking into consideration those factors and improves the performance in terms of energy efficiency and network lifetime. Very diminutive work is done on the comparison of QoS parameters of EEPB and IEEPB. Parameters such as Delay, throughput, Energy consumption and packet drop ratio can further be analyzed and compared. Improvisations on these parameters can also be performed to recuperate the end to end performance of IEEPB protocol.

\section{References}

[1]. J. Al-Karaki, and A. Kamal, .Routing Techniques in Wireless Sensor Networks: A Survey., IEEE Communications Magazine, vol 11 , no. 6, pp. 6-28, Dec. 2004

[2]. Wireless Sensor Networks. (2014,March,20). MDPI[Online].Available: www.mdpi.com/journal/sensors

[3]. S. Singh, M. Singh, D. Singh, "A Survey of Energy-Efficient Hierarchical Cluster-Based Routing in Wireless Sensor Networks", Int. J. of Advanced Networking and Applications Volume: 02, Issue: 02, Pages: 570-580, 2010.

[4]. W.R. Heinzelman, A. Chandrakasan, and H. Balakrishnan, .Energy-efficient Communication Protocol for Wireless Microsensor Networks., in IEEE Computer Society Proceedings of the Thirty ThirdHawaii International Conference on System Sciences(HICSS '00), Washington DC, USA, , vol. 8, pp. 8020, Jan. 2000.

[5]. Wireless Sensor Networks. (2013,December,16). Mdpi [Online]. Available http://www.nhu.edu.tw/ cmwu/Lab/Routing\%20protocol\%20on\%20wireless\%20sensor\%20network.ppt.

[6]. Lindsey, S.; Raghavendra, C.S. PEGASIS: Power-Efficient Gathering in Sensor Information Systems. In Proceedings of the Aerospace Conference, Big Sky, MT, pp. 1125-1130, March, 2002.

[7]. Liu Yueyang, Ji Hong and YueGuangxin, "An Energy-Efficient PEGASIS-Based Enhanced Algorithm in WirelessSensorNetworks", Technology Forum, China Communications, August 2006.

[8]. GUO. Wen-yu, ZHANG Wei and LU Gang, "PEGASIS protocol in wireless sensor network based on an improved ant colony algorithm," in the IEEE proceedings of Second International Workshop on Education Technology and Computer Science, pp.6467,2010 . 
[9]. WANG Lin-ping, BI Wu, CAI Zhen and WANG Zu-feng, "Improvedalgorithm of PEGASIS protocol introducing double cluster heads inwireless sensor network," International Conference onComputer,Mechatronics,Control and Electronic Engineering, 2010,pp.148-151.

[10]. FengSen, Qi Bing, Tang Liangrui, “An Improved Energy-Efficient PEGASIS-Based Protocol in Wireless Sensor Networks”, IEEE Eighth International Conference on Fuzzy Systems and Knowledge Discovery, pp.2230-2233, 2011.

[11]. Vhatkar.S, M.Atique, "Design Issues, Characteristics and Challenges in Routing Protocols for Wireless Sensor Networks", International Journal of Computer Applications (0975 - 8887) International Conference and Workshop on Emerging Trends in Technology 2013.

[12]. http://hscc.cs.nthu.edu.tw/ sheujp/lecture_note/13wsn/ppt/WSN_Chapter\%204\%20Routing\%20Protocols-I.pptx

[13]. http://www.google.com/patents/US8295280. 